\title{
Inflammatory cytokines and experimental arterial and vein grafts
}

\author{
Antonio V. Sterpetti, MD, Paolo Sapienza, MD, Valeria Borrelli, PhD, and Luca Di Marzo, MD
}

From the University of Rome Sapienza, Rome, Italy.
This work was supported by a grant from University of Rome.
Disclosures: Authors have nothing to disclose with regard to commercial support.
Received for publication Nov 5, 2019 ; revisions received Nov 5, 2019; accepted for publication Jan 3, 2020;
available ahead of print Feb 4, 2020 .
Address for reprints: Antonio V. Sterpetti, MD, Viale del Policlinico 00167, Rome, Italy (E-mail: antonio.
sterpetti @ uniroma1.it).
JTCVS Techniques 2020;1:48-50
2666-2507
Copyright @ 2020 The Author(s). Published by Elsevier Inc. on behalf of The American Association for Thoracic
Surgery. This is an open access article under the CC BY-NC-ND license (http://creativecommons.org/licenses/by-
nc-nd/4.0/).
https://doi.org/10.1016/j.xjtc.2020.01.008

Ten years after surgery, only $40 \%$ of the saphenous coronary artery bypass grafts have a normal appearance. ${ }^{1}$ Myointimal hyperplasia and atherosclerosis are unusual in the internal thoracic artery. A systematic review has shown better patency rates for the radial artery. ${ }^{2}$ The aim of our study was to determine the release of several inflammatory cytokines from experimental arterial and vein grafts. Increased production of inflammatory cytokines by vein grafts might lead to higher incidence of occlusive changes.

\section{MATERIAL AND METHODS \\ Study Design}

Male Lewis rats were used for the experiments. In 80 animals, a 1-cmlong segment of vena cava, explanted from syngeneic Lewis rats, was inserted at the level of the abdominal aorta. In 16 animals, the arterial vein graft was explanted 4 weeks from surgery and reimplanted in the iliac vein of syngeneic Lewis rats. The reimplanted grafts were harvested 2 weeks and 8 weeks later.

In another 20 male Lewis rats, a 1-cm-long segment of abdominal aorta, obtained from syngeneic Lewis rats, was inserted at the level of the abdominal aorta. Four weeks after surgery, the grafts were removed. All explanted grafts were analyzed for scanning and electron microscopy, histochemistry, and inflammatory cytokine release in an organ culture (Appendix 1).

\section{RESULTS}

\section{Lumen Diameter and Intima-Media Thickness}

The lumen diameter and thickness of the wall increased in veins implanted into the arterial circulation $(P<.0001)$. There was no significant further increase 4 weeks after surgery (Figure 1). Once reimplanted in the venous circulation, the intima-media layer and the lumen diameter decreased significantly $(P<.001)$. Eight weeks after reimplantation, the area of myointimal hyperplasia disappeared completely. In arterial grafts, there was no evidence of myointimal hyperplasia or changes in lumen diameter.

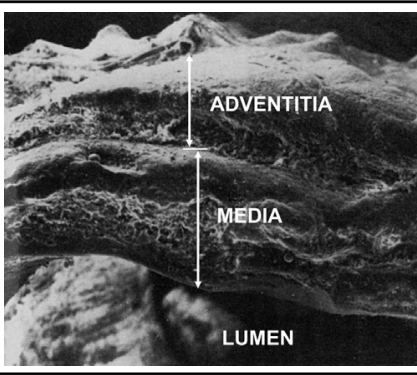

Electron scanning. Transverse section of a vein graft 4 weeks after implantation into the arterial system $(\times 20)$. Thickening of the wall of vein grafts is a physiologic adaptation to the arterial system regulated by a delicate balance of many factors. This balance can be altered in specific hemodynamic conditions, and it may be related to genetic factors and aging through increased production of inflammatory cytokines.

CENTRAL MESSAGE

Thickening of the wall of vein grafts is a physiologic adaptation to the arterial system, regulated by a delicate balance of many factors. This balance can be altered in specific hemodynamic conditions, and it may be related to genetic factors and aging through increased production of inflammatory cytokines.

See Commentaries on pages 51 and 53 .

\section{Histology}

The area of myointimal hyperplasia in arterial vein grafts consisted mainly of smooth muscle cells. The adventitia presented significant thickening, with predominance of collagen deposition. All these changes regressed once the arterialized vein graft was reimplanted in the venous circulation. Arterial grafts did not show significant differences in comparison with the preimplantation histology. Histochemistry demonstrated that endothelial cells and adventitial cells of arterial vein grafts were positive for interleukin (IL)-1, IL-2, and IL-6. 


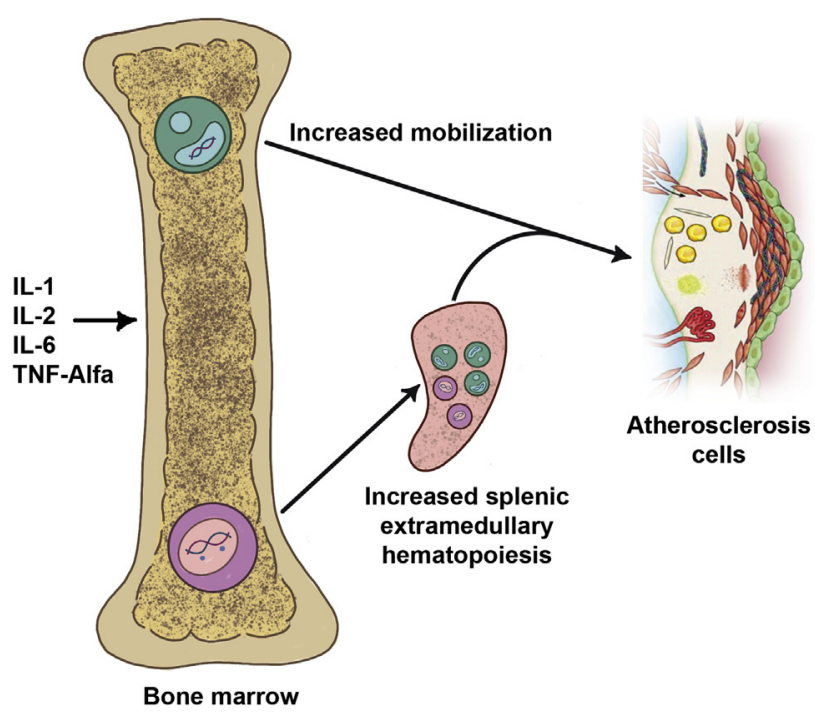

FIGURE 1. Schematic drawing representing the possible interactions between the inflammatory cytokines produced by the veins grafts and deregulation of the immune system. The condition of chronic inflammation, determined by increased local production of inflammatory cytokines by arterial vein grafts or other sources can determine a central and systemic deregulation of the immune system. This deregulation can directly influence an increased production of inflammatory cytokines in the periphery, leading to chronic inflammation and progression of myointimal hyperplasia to atherosclerotic plaque, eventually leading to graft occlusion. $I L$, Interleukin; $T N F$, tumor necrosis factor.

\section{Analysis of Graft-Conditioned Media}

In organ culture, arterial vein grafts released an increased, time-dependent amount of inflammatory cytokines $(P<.001)$. This increased production was not present in arterial grafts (Tables 1 and 2).

\section{DISCUSSION}

The vein adapts to the arterial circulation, increasing the internal diameter and the thickness of the wall to respond to higher internal pressure and larger quantities of blood. This adaptive changes include reduced compliance.

Vein grafts may undergo occlusive changes. The abnormal segmental hyper-plastic reaction that can progress to atherosclerotic plaque may be related to turbulences occurring near the valves or in fibrotic segments. Inflammatory cytokines may play a major role in the progression from myointimal hyperplasia to atherosclerotic plaque, inducing activation of leukocytes and macrophages, proliferation of smooth muscle cells, and transport of lipids into the plaque. These actions determine increasing size of the plaque transforming a simple plaque in a "dangerous" unstable plaque.,

An aggressive antiplatelet and lipid-lowering therapy has been shown to improve patency rates of coronary vein grafts. It is possible that those therapies could have a major role in reducing the production and effect of inflammatory cytokines.

New targeted drugs, with a more selective action, have been developed and applied in the clinical setting. The results of the CANTOS trial ${ }^{5}$ show that inhibition of IL-1 beta in patients with previous myocardial infarction and increased levels of inflammatory markers reduces the risk for new cardiovascular events.

We reviewed the clinical outcomes of 2000 autologous vein grafts implanted into the arterial circulation of children. Graft occlusion due to abnormal formation of myointimal hyperplasia in the graft was rare. Ageing per se might facilitate atherosclerosis. Inflammatory cytokines can exert their action locally and systemically. Chronic inflammation might deregulate with aging of the central immune system, activating specific clones, with an autoimmune action.

The vein adapts to the arterial circulation through complex mechanisms in a delicate balance that can be altered in abnormal hemodynamic conditions, genetic factors, and aging. The artery implanted as autologous graft does not face such an adaptation.

\section{CONCLUSIONS}

Future studies are needed to investigate possible methods to reduce occlusion in coronary vein grafts. It is essential to distinguish between physiologic adaptation of the vein graft to the arterial circulation from the pathological changes that are related to specific abnormal flow hemodynamics, advanced age of the patient, and systemic factors.

TABLE 1. Inflammatory cytokine release by vein grafts implanted in the arterial circulation and by vein grafts implanted for 4 weeks in the arterial circulation and reimplanted in the venous system

\begin{tabular}{lccccc}
\hline $\mathbf{n g} / \mathbf{c m}^{2}$ & Control vein & AVG 7 d & AVG 4 wk & RV 2 wk & RV 8 wk \\
\hline IL-1 & $0.2 \pm 0.05$ & $3.6 \pm 0.9$ & $3.8 \pm 0$ & $0.5 \pm 011$ & $0.3 \pm 0.1$ \\
IL-2 & $0.2 \pm 0.03$ & $4.0 \pm 0.8$ & $4.2 \pm 0.3$ & $0.4 \pm 0.1$ & $0.3 \pm 0.05$ \\
IL-6 & $0.01 \pm 0.01$ & $3.9 \pm 0.2$ & $4.0 \pm 0$ & $0.6 \pm 0.05$ & $0.2 \pm 0.1$ \\
TNF- $\alpha$ & $24 \pm 2$ & $33 \pm 3$ & $50 \pm 6$ & $220 \pm 15$ & $23 \pm 2.1$ \\
\hline
\end{tabular}

$A V G$, Arterialez vein grafts; $R V$, reimplanted vein grafts; $I L$, interleukin; $T N F$, tumor necrosis factor. 
TABLE 2. Inflammatory cytokine release by arterial grafts explanted and reimplanted in the arterial circulation

\begin{tabular}{lcc}
\hline $\mathbf{n g} / \mathbf{c m}^{\mathbf{2}}$ & Preimplantation & Postimplantation (4 wk) \\
\hline $\mathrm{IL}-1$ & $0.3 \pm 0.05$ & $0.4 \pm 0.9$ \\
$\mathrm{IL}-2$ & $0.4 \pm 0.1$ & $0.6 \pm 0.2$ \\
$\mathrm{IL}-6$ & $0.1 \pm 0.1$ & $1.2 \pm 0.2$ \\
\hline
\end{tabular}

IL, Interleukin.

\section{References}

1. de Vries MR, Simons KH, Jukema JW, Braun J, Quax PH. Vein graft failure: from pathophysiology to clinical outcomes. Nat Rev Cardiol. 2016;13:451-70.

2. Gaudino M, Benedetto U, Fremes S, Biondi-Zoccai G, Sedrakyan A, Puskas JD, et al. Radial-artery or saphenous-vein grafts in coronary-artery bypass surgery. N Engl J Med. 2018;378:2069-77.

3. Sterpetti AV, Cucina A, Borrelli V, Ventura M. Inflammation and myointimal hyperplasia: correlation with hemodynamic forces. Vasc Pharm. 2019;117:1-6.

4. Sterpetti AV, Lepidi S, Borrelli V, Di Marzo L, Sapienza P, Cucina A, et al. Growth factors and experimental arterial grafts. J Vasc Surg. 2016;64:1444-9.

5. Ridker PM, Everett BM, Thuren T, MacFadyen JG, Chang WH, Ballantyne C, et al. CANTOS Trial Group. Antiinflammatory therapy with canakinumab for atherosclerotic disease. $N$ Engl J Med. 2017;377:1119-31.

\section{APPENDIX 1. SURGICAL TECHNIQUES}

The thoracic inferior vena cava was used as graft. It was implanted in the infrarenal abdominal aorta of Lewis rats in an end-to-end fashion; 10-0 nylon sutures were used (Ethicon Inc, Somerville, NJ) in a continuous fashion, with 2 starting sutures placed at $180^{\circ}$.

In 16 animals, the graft was harvested 4 weeks after being in the arterial circulation and reimplanted at the level of the left iliac vein of recipient Lewis rats, with the same technique as described earlier.

At harvest, the grafts were opened in the middle to assess the patency, checking the inflow and the outflow. All grafts were patent at the time of sacrifice. No antiaggregant or anticoagulation was used.
In another 20 male Lewis rats, a 1-cm-long segment of abdominal aorta, obtained from syngeneic Lewis rats, was inserted at the level of the abdominal aorta. Four weeks after surgery, the grafts were removed and analyzed for scanning and electron microscopy, histochemistry, and cytokine release. Animal care complied with the Guide for the Care and Use of Laboratory Animals (US National Institutes of Health, N. 80-23, modified 1996). Xylazine and ketamine were used for anesthesia. Animals were humanely killed with an overdose of anesthetic. The study was approved by the Department Council and the Laboratory Council.

\section{Explanted Grafts Analysis}

Grafts organ culture. The technique has been described. In brief, the graft specimens were washed with Dulbecco Modified Eagle Medium with antibiotics and placed in culture plates (Costar, Cambridge, Mass). The graft specimens were put in the organ culture at a $\mathrm{pH}$ of $7.4,37^{\circ} \mathrm{C}$, and $5 \%$ $\mathrm{CO} 2$ atmosphere. Aliquots of the culture media were taken at 72 hours, and the concentrations of the inflammatory cytokines were analyzed. The specimens were viable after 72 hours of incubation by cytofluorometry and titrated thymidine uptake.

Determination of inflammatory cytokines in the conditioned media. These substances were determined quantitatively in the grafts' conditioned media by inhibition of antibody-binding assay with a method previously described. ${ }^{3}$ In brief, antibodies to the different substances were added. The antibody-binding activity was measured, after further steps, by enzyme-linked immunosorbent assay. Bound specific antibody was measured by optical density reading at $492 \mathrm{~nm}$ with a spectrophotometer. The quantity of the different substances was assessed using known quantities of that substance. 